\title{
Validating the Patient Experience with Treatment and Self-Management (PETS), a patient-reported measure of treatment burden, in people with diabetes
}

\author{
Elizabeth A Rogers ${ }^{1,2}$ \\ Kathleen J Yost ${ }^{3}$ \\ Jordan K Rosedahl ${ }^{3}$ \\ Mark Linzer ${ }^{4}$ \\ Deborah H Boehm ${ }^{5}$ \\ Azra Thakur ${ }^{5}$ \\ Sara Poplau ${ }^{5}$ \\ Roger T Anderson ${ }^{6}$ \\ David T Eton ${ }^{3}$ \\ 'Department of Medicine, University \\ of Minnesota Medical School, \\ Minneapolis, MN, USA; ${ }^{2}$ Department \\ of Pediatrics, University of Minnesota \\ Medical School, Minneapolis, MN, \\ USA; ${ }^{3}$ Department of Health Services \\ Research, Mayo Clinic, Rochester, \\ MN, USA; ${ }^{4}$ Department of Medicine, \\ Hennepin County Medical Center, \\ Minneapolis, MN, USA; ${ }^{5}$ Minneapolis \\ Medical Research Foundation, \\ Minneapolis, MN, USA; ${ }^{\circ}$ University \\ of Virginia School of Medicine, \\ Charlottesville, VA, USA
}

This article was published in the following Dove Press journal: Patient Related Outcome Measures

9 November 2017

Number of times this article has been viewed

Aims: To validate a comprehensive general measure of treatment burden, the Patient Experience with Treatment and Self-Management (PETS), in people with diabetes.

Methods: We conducted a secondary analysis of a cross-sectional survey study with 120 people diagnosed with type 1 or type 2 diabetes and at least one additional chronic illness. Surveys included established patient-reported outcome measures and a 48-item version of the PETS, a new measure comprised of multi-item scales assessing the burden of chronic illness treatment and self-care as it relates to nine domains: medical information, medications, medical appointments, monitoring health, interpersonal challenges, health care expenses, difficulty with health care services, role activity limitations, and physical/mental exhaustion from self-management. Internal reliability of PETS scales was determined using Cronbach's alpha. Construct validity was determined through correlation of PETS scores with established measures (measures of chronic condition distress, medication satisfaction, self-efficacy, and global well-being), and known-groups validity through comparisons of PETS scores across clinically distinct groups. In an exploratory test of predictive validity, step-wise regressions were used to determine which PETS scales were most associated with outcomes of chronic condition distress, overall physical and mental health, and medication adherence.

Results: Respondents were 37-88 years old, 59\% female, 29\% non-white, and 67\% collegeeducated. PETS scales showed good reliability (Cronbach's alphas $\geq 0.74$ ). Higher PETS scale scores (greater treatment burden) were correlated with more chronic condition distress, less medication convenience, lower self-efficacy, and worse general physical and mental health. Participants less (versus more) adherent to medications and those with more (versus fewer) health care financial difficulties had higher mean PETS scores. Medication burden was the scale that was most consistently associated with well-being and patient-reported adherence.

Conclusion: The PETS is a reliable and valid measure for assessing perceived treatment burden in people coping with diabetes.

Keywords: treatment burden, patient-reported measure, measurement, patient perspective, disease management

\section{Introduction}

Diabetes management is burdensome. It demands that individuals engage in care, perform self-monitoring, manage medications, seek health information, change their lifestyle, and navigate specialty care. It is also heterogeneous. For adults with type 2 diabetes (e.g., insulin-resistant diabetes), self-management can range from monitoring 
diet and exercise and taking one or two pills a day, to intensive pill and injectable insulin regimens that require dosing three or four times a day along with glucose self-monitoring and attention to diet and exercise. This intensive end of the self-management spectrum mirrors management of type 1 diabetes (e.g., autoimmune diabetes that requires insulin). In addition to diabetes management, nearly $90 \%$ of primary care patients with type 2 diabetes are also managing at least one additional chronic condition. ${ }^{1}$ Some comorbid conditions may have care goals that overlap with diabetes (e.g., hypertension, hyperlipidemia) while others' care goals do not (e.g., asthma, osteoarthritis), adding to the heterogeneity of patient experience with self-management. ${ }^{2}$ This has the potential to compound a person's sense of treatment burden, defined as the perceived "workload" of health care and its impact on a person's functioning and well-being. ${ }^{3-5}$

Among people with multiple chronic conditions, and after adjusting for age, number of chronic conditions, and presence of an unpaid carer, diabetes and other endocrine conditions were the only chronic conditions independently associated with a higher likelihood of experiencing treatment burden. ${ }^{6}$ Greater treatment burden is associated with higher hemoglobin A1c levels and poorer adherence to self-care, ${ }^{3}$ and may worsen health-related quality of life..$^{7-9}$ Clinicians focused on meeting care quality standards and guidelines may respond to these poor outcomes by escalating treatment, requiring more from the patient, such as taking more medications, more frequent self-monitoring, and greater lifestyle restrictions. Asking patients to do more risks a "downward spiral" of diminished adherence to self-management, worse mental and physical health, and ultimately poorer patientperceived quality of care. ${ }^{10}$ In these cases, the patient-centric perspective of care appears to have been lost.

The American Diabetes Association (ADA) recommends psychosocial assessment as a critical part of patient-centered care in order to promote optimal medical outcomes and psychosocial well-being. ${ }^{11}$ The European Society of Cardiology, in collaboration with the European Association for the Study of Diabetes, also recommends patient-centered care and consideration of the context of patient priorities and goals. ${ }^{12}$ Understanding treatment burden is an important part of providing patient-centered care by making explicit the psychosocial and behavioral challenges faced by people with chronic illnesses who require self-management. Several existing patient-reported measures in diabetes include assessment of some aspects of treatment burden. One review identified 47 diabetes-specific measures which contain domains indicative of treatment burden, including treatment and self-care convenience, medication burden, monitoring burden, economic burden, medical device burden, family conflict/strain, lifestyle impact, and regimen-related distress. ${ }^{3}$ Most of these scales were not designed to be comprehensive measures of treatment burden per se. As such, they may assess some, but not all of the important elements of this multi-dimensional construct. Furthermore, because "diabetes-specific" measures assess diabetes-specific effects, they may be less sensitive at addressing issues of concern to people who have diabetes co-existent with other burdensome chronic conditions that require self-management (e.g., kidney, cardiovascular, and pulmonary disease). Accurate assessment of the burden issues of this population would require use of multiple, disease-specific measures. A general measure that comprehensively assesses aspects of treatment and self-management burden common to many conditions would therefore be a valuable tool, one that could help fill this existing gap in measurement.

We recently developed and validated a comprehensive patient-reported measure of treatment burden, the Patient Experience with Treatment and Self-Management (PETS) questionnaire in people with multiple chronic conditions. ${ }^{13}$ In this secondary analysis of data collected as part of the previous validation study, we will assess the reliability and validity of the PETS in people managing diabetes using a subsample of participants with a diagnosis of diabetes (type 1 or 2) who come from the original validation study cohort of people with multiple chronic conditions.

\section{Subjects, materials, and methods The PETS measure}

Informed by a previously generated conceptual framework derived from interviews and focus groups with patients with multiple chronic conditions, ${ }^{5}$ and supported by initial psychometric analyses including a confirmatory factor analysis, ${ }^{13}$ the current version of the PETS features nine multi-item domain scales. These nine scales measure the burden of chronic illness treatment as it relates to: medical information (seven items), medications (seven items), medical appointments (three items), monitoring health (two items), interpersonal challenges (four items), health care expenses (five items), difficulty with health care services (seven items), role/social activity limitations due to self-management (six items), and physical/mental exhaustion due to self-management (five items). Two individual items indicative of distress with taking medication - "bother" due to reliance on medicines and "bother" due to side effects of medicines - are scored as single-item indicators. Hence, 11 domain scores are available 
on this version of the PETS (nine multi-item + two singleitem scales). Items use either a 4- or 5-point Likert response scale and a 4-week recall time frame. A "not applicable" response option is included for select items that address issues of relevance to only certain respondents. Domain scales include questions such as, "How much of a problem has it been for you to organize your medicines?" (medication burden); "How easy or difficult has it been for you to understand any changes to your treatment plan?" (medical information); "How much of a problem has it been for you to schedule and keep track of your medical appointments?" (medical appointments); "How much of a problem has it been for you to monitor your health condition, for example, weighing yourself, checking your blood pressure, or checking your blood sugar?" (monitoring health); and "How much has your self-management interfered with your ability to spend time with family and friends?" (role and social activity limitations). Each domain scale is scored separately, and users are free to choose those domains applicable to their setting and context. A single factor structure has not been supported, hence there is no total PETS score. ${ }^{13}$ A complete report of the development and initial validation of the PETS in people with multiple chronic conditions can be found in Eton et al. ${ }^{13}$ This report includes full details on the development of the initial item pool, item reduction and modification, determination of factor structure (i.e., through confirmatory factor analysis), scale scoring, and validation of the measure. The 48-item version of the measure validated in the prior study and used in this study can be found in Figure S1.

\section{Sample}

Participants were recruited from two clinical sites: the Hennepin County Medical Center (HCMC, Minneapolis, MN, USA) and the Mayo Clinic (Rochester, MN, USA). HCMC is a public "safety net" hospital that provides care for low-income, uninsured, and vulnerable persons in urban Minneapolis. The Mayo Clinic is a large multi-specialty integrated practice located in southeast Minnesota. Participants were eligible to be surveyed in the parent validation study if they: 1) were $\geq 21$ years old, 2) were assigned to a primary care provider at either site, 3 ) had medical recordconfirmed diagnoses of two or more chronic conditions (specifically conditions requiring burdensome treatment and/or self-management strategies), ${ }^{14-17}$ 4) had at least one medical record-confirmed encounter with a provider at either site within the past 18 months for one or more of the selected chronic conditions, and 5) were proficient in the English language. The subsample used in this secondary analysis to validate the PETS measure in people with diabetes consisted of those participants who returned completed surveys and had a diagnosis of type 1 or type 2 diabetes (ICD- 9 codes 250.00-250.93). This included 120 of the original 332 multi-morbid participants who returned completed surveys. Consistent with the initial eligibility criteria, participants had at least one other comorbid condition, including those that frequently co-occur with diabetes (e.g., hypertension, hypercholesterolemia, coronary artery disease, and chronic kidney disease).

\section{Procedure}

The Mayo Clinic Survey Research Center prepared the survey battery for mailing. The battery consisted of the 48-item PETS measure as well as several established scales. A 5-item chronic condition distress scale, adapted from the Diabetes Distress Scale $^{18}$ and specified generally for "health problems" (L Fisher, personal communication, July, 2014), was used to assess overall distress associated with living with a chronic health condition. Sample items include feeling overwhelmed by my health problems, feeling that my health care team is not giving me enough help with how to self-manage my health problems, and feeling that my chronic health condition kept me from doing the things that I like to do. The 5-item Side Effects and 3-item Convenience subscales of the Treatment Satisfaction Questionnaire for Medication (TSQM) ${ }^{19}$ were used to assess trouble with medication side effects and the convenience of taking required medications, respectively. The 8-item Perceived Medical Condition Self-Management scale (PMCSM) was used to provide a general dispositional assessment of selfefficacy or perceived competence in managing one's health condition. ${ }^{20}$ Finally, the Patient-Reported Outcomes Measurement Information System (PROMIS) Global-10 was used to assess global physical health (GPH) and global mental health $(\mathrm{GMH}) .{ }^{21}$ Validity and reliability of each of these scales has been demonstrated in people with chronic illnesses, and they have been used previously in people with diabetes. ${ }^{18-23}$

Age, race/ethnicity, education level, work status, personal health-related issues (i.e., number of prescription medications, financial difficulties due to medical care), and other health-relevant concepts (i.e., medication adherence, convenience of health care services) $)^{24-26}$ were assessed using targeted single items. Gender, chronic conditions, and encounters with a medical provider in the last 18 months were extracted from the electronic medical record.

Survey mailing consisted of the instrument battery, a cover letter, a small gift to encourage participation (designer pen or $\$ 3$ gift card), and a postage-paid return envelope. The 
survey was entirely self-administered. A second mailing of the survey to non-respondents occurred 3 weeks after the first mailing. The period of recruitment was September through November of 2014.

The study was deemed exempt by both site Institutional Review Boards under category 2 of Title 45 Code of Federal Regulations (CFR) Part 46.101 (Protection of Human Subjects). This states that a study is eligible for category 2 exempt status if: 1) it does not involve prisoners, 2) it does not involve an approved US Food and Drug Administration regulated product used in the course of medical practice, 3 ) it is research that involves only the use of educational tests or survey procedures with i) the information obtained recorded in such a manner that human subjects cannot be identified, either directly or through identifiers, and ii) any disclosure of human subjects' responses outside the research would not reasonably place the subjects at risk of criminal or civil liability or be damaging to their financial standing, employability, or reputation. The current research did not involve procedures that require written consent outside of the research context (i.e., the surveys), and all criteria for waiver of consent documentation were met in accordance with 45 CFR 46.117. Therefore, waiver of consent documentation was approved. The cover letter described the study, explained how data would be aggregated for analysis and kept secure, and clarified that participants could skip any questions they did not want to answer. Participant anonymity was preserved by the following: 1) names and addresses were only recorded to create mailing address labels, 2) a unique study identifier was used to track survey returns and to link with medical record data, 3) returned survey data were pooled across all participants and only an aggregated dataset was analyzed, and 4) the file containing participant names and addresses was destroyed at the conclusion of the study.

\section{Analyses}

\section{Scoring the PETS}

After reverse-coding of positively worded items, unweighted item scores were summed to form raw scale scores. Given that some items address issues that may not be applicable to all respondents, the scale scores were prorated for missing item data, as long as more than $50 \%$ of the items within a scale were answered. Items answered as "not applicable" as well as those that were un-answered were treated as missing. The prorated raw scale score is obtained using the following formula: [(sum of the item scores in the scale that are answered or non-missing) $\times$ (total number of items in the scale) $] /($ number of items in the scale that are answered or non-missing). This form of scoring handles missing data by replacing any un-answered items within a scale with the mean of the answered items of the scale as long as more than $50 \%$ are completed. This approach to scoring has been found to reduce bias and enhance precision of multi-item scales. ${ }^{27}$ When more than $50 \%$ of the items within a scale are missing, the entire domain scale is treated as missing. Raw domain scores were then transformed to a standardized 0 to 100 metric, with a higher score indicative of greater treatment burden.

\section{Reliability and validity}

Cronbach's alphas were computed for the PETS domain scales to determine internal consistency reliability. Alphas $\geq 0.70$ indicate adequate reliability. ${ }^{28}$ Spearman correlations (rho) were used to determine convergent validity through associations between PETS domain scores and scores from established measures of conceptually related constructs. We hypothesized that higher PETS scores would be correlated with greater chronic condition distress (Chronic Condition Distress scale), greater bother of medication side effects (TSQM side effects), less medication convenience (TSQM convenience), lower self-efficacy for managing chronic illness (PMCSM), poorer overall physical and mental health (PROMIS-10), and lower self-rated convenience of health care services. Correlations of at least moderate magnitude (rho $\geq 0.30$ ) and in the hypothesized directions support validity. ${ }^{29}$ Correlations with data extracted from the medical record were also determined to assess validity of PETS scores in people with diabetes. We hypothesized that having more comorbid conditions and having more numerous recent encounters with medical providers would be associated with higher PETS scores (i.e., more burden). As in the previous validation study, independent samples $t$-tests were used to compare mean PETS scores across meaningful and distinct participant groups that are likely to differ in perceived treatment burden (i.e., known-groups validity). ${ }^{13}$ For these comparisons, we hypothesized that participants self-reporting lower adherence to required medications and more financial difficulties because of their physical condition or medical care would have higher burden scores. Cohen's $d$ was calculated and used to indicate the effect sizes by dividing each group difference in PETS score by the pooled standard deviation, with $d=0.2$, 0.5 , and 0.8 considered small, medium, and large effect sizes, respectively. ${ }^{29}$ Given the large number of analyses, a relatively conservative alpha level of 0.01 was set for all correlations and group comparisons. 


\section{Exploratory regression analyses of outcomes associated with PETS scores}

To explore potential predictive validity of the PETS, we conducted a series of step-wise regression analyses to determine whether certain PETS domain scores are more highly associated with meaningful patient outcomes including distress, physical and mental health, and adherence to required medications. Multiple linear regressions were used to model potential predictors of continuous outcome variables (i.e., chronic condition distress, overall physical health, and overall mental health) and a logistic regression was used to model potential predictors of the one binary outcome variable (i.e., medication adherence). In each regression the number of comorbid conditions and the number of provider encounters in the last 18 months were initially controlled for through forced entry into each model at step 1. A forward selection procedure was then used to select those PETS scales most associated with each outcome, with the $p$-value for variable entry set at 0.05 . For linear regressions, the strength of association between each entered variable and the continuous outcome, relative to other variables in the model, is indicated by the standardized regression weight (standardized beta) and changes in the variance explained at each step $\left(\Delta \mathrm{R}^{2}\right)$. Fit of the final model featuring all significant variables entered is indicated by the overall model $\mathrm{R}^{2}$ (i.e., total amount of variance in the outcome explained by all entered variables, ranging from 0 to 1 ). For the logistic regression, the strength of association between each entered variable and the dichotomous outcome, relative to other variables in the model, is indicated by odds ratios along with their associated $95 \%$ confidence intervals. Overall model fit with all significant variables entered is indicated by the final model chi-square. No hypotheses are proposed for this exploratory analysis. All statistical analyses were conducted in SPSS Statistics version $20^{\circledR}$ (IBM Corporation, Armonk, NY, USA).

\section{Results}

\section{Sample characteristics}

Demographic, medical, and other health-related characteristics of the sample are identified in Table 1. There were slightly more women (59\%) than men, with a mean age of approximately 64 years (range: $37-88$ ). Most identified their race as white $(66 \%)$, were married or living with a partner $(53 \%)$, and had at least some formal college or university education (65\%). From the electronic medical record, the median number of diagnosed conditions was four and the median number of encounters with a health care provider in the past 18 months was 12 . The most common comorbidities
Table I Demographic, medical, and other health-related characteristics of survey respondents

\begin{tabular}{|c|c|}
\hline Characteristics of respondents & $\mathrm{N}=120 *$ \\
\hline Age (years), mean (range) & $63.9(37-88)$ \\
\hline \multicolumn{2}{|l|}{ Gender, N (\%) } \\
\hline Female & $71(59)$ \\
\hline Male & $49(4 I)$ \\
\hline \multicolumn{2}{|l|}{ Race, $\mathbf{N}(\%)$} \\
\hline White & $79(66)$ \\
\hline Black/African-American & $22(18)$ \\
\hline Asian & $6(5)$ \\
\hline Mixed & $4(3)$ \\
\hline Native American/American Indian & $3(3)$ \\
\hline Hispanic/Latino ethnicity, $N(\%)$ & $6(5)$ \\
\hline \multicolumn{2}{|l|}{ Marital status, $\mathbf{N}(\%)$} \\
\hline Married or living with partner & $63(53)$ \\
\hline Not married & $53(44)$ \\
\hline \multicolumn{2}{|l|}{ Education level, $\mathbf{N}(\%)$} \\
\hline Less than High school & $12(10)$ \\
\hline High school graduate & $27(23)$ \\
\hline Some college/Associate's degree & $46(38)$ \\
\hline College graduate (B.A., B.S.) & $20(17)$ \\
\hline Graduate school/Advanced degree & $12(10)$ \\
\hline \multicolumn{2}{|l|}{ Work status, $\mathbf{N}(\%)$} \\
\hline Not working & $80(67)$ \\
\hline Working full or part-time & $36(30)$ \\
\hline \multicolumn{2}{|l|}{ No. of other comorbid conditions, $\mathbf{N}(\%)$} \\
\hline 2 & $9(8)$ \\
\hline 3 & $42(35)$ \\
\hline 4 or more & $69(58)$ \\
\hline Median no. of conditions & 4.0 \\
\hline \multicolumn{2}{|l|}{ Other comorbid conditions, N (\%) } \\
\hline Hypertension & $102(85)$ \\
\hline Disorders of lipid metabolism & $93(78)$ \\
\hline Osteoarthritis & $38(32)$ \\
\hline Chronic kidney disease & $27(23)$ \\
\hline Coronary artery disease & $19(16)$ \\
\hline Asthma & $17(14)$ \\
\hline Depression & $14(12)$ \\
\hline Chronic obstructive pulmonary disease & $14(12)$ \\
\hline Glaucoma & $10(8)$ \\
\hline Hepatitis (B and C) & $7(6)$ \\
\hline \multicolumn{2}{|l|}{ No. provider encounters in last 18 months, $\mathbf{N}(\%)$} \\
\hline $\mathrm{I}-8$ & $45(38)$ \\
\hline $9-17$ & $34(28)$ \\
\hline $18+$ & $4 I(34)$ \\
\hline Median no. of encounters & 12.0 \\
\hline \multicolumn{2}{|l|}{ Taking prescription medications, $\mathbf{N}(\%)$} \\
\hline Yes & $112(93)$ \\
\hline No & $\mathrm{I}(\mathrm{I})$ \\
\hline \multicolumn{2}{|l|}{ No. of prescription medications, $\mathbf{N}(\%)$} \\
\hline 1 & $2(2)$ \\
\hline $2-3$ & $17(14)$ \\
\hline $4-5$ & $31(26)$ \\
\hline 6 or more & $56(47)$ \\
\hline \multicolumn{2}{|l|}{ Adherence to medications, $\mathbf{N}(\%)$} \\
\hline Always take all medications & $89(80)$ \\
\hline $\begin{array}{l}\text { Usually or sometimes take all medications }(<80 \% \\
\text { of time) }\end{array}$ & $22(20)$ \\
\hline
\end{tabular}

(Continued) 
Table I (Continued)

\begin{tabular}{ll}
\hline Characteristics of respondents & $\mathbf{N}=\mathbf{I} \mathbf{2 0} *$ \\
\hline Any health insurance, $\mathbf{N}(\%)$ & $101(84)$ \\
Yes & $13(\mathrm{II})$ \\
No & $2(2)$ \\
Not sure & \\
Health care causing financial difficulties, $\mathbf{N}(\%)$ & $4 I(34)$ \\
Not at all & $39(33)$ \\
A little & $36(30)$ \\
Somewhat or more &
\end{tabular}

Notes: *Due to missing values, $\mathrm{N}=$ I |4 for race; $\mathrm{N}=1$ | 6 for marital status, work status, any health insurance, and health care causing financial difficulties; $\mathrm{N}=117$ for education level; $\mathrm{N}=1$ I 3 for taking prescription medications; $\mathrm{N}=106$ for no. of prescription medications; and $\mathrm{N}=\mathrm{I}$ I I for adherence to medications.

included hypertension (85\%), lipid metabolic disorders (78\%), osteoarthritis (32\%), chronic kidney disease (23\%), and coronary artery disease (16\%).

\section{PETS characteristics and reliability}

Mean PETS scores, score ranges obtained, and internal consistency reliability for all PETS multi-item scales are shown in Table 2. Mean PETS scores were slightly skewed in the direction of less burden (lower domain score). All nine of the multi-item PETS scales showed good internal consistency (reliability). Cronbach's alpha coefficients were well above threshold for adequate reliability (alpha $\geq 0.70$ ). ${ }^{28}$

\section{Convergence of PETS scores with other measures}

As shown in Table 3, PETS domain scales were significantly correlated with many of the established measures of related constructs. Higher PETS scores (i.e., more burden) were associated with greater chronic condition distress (Chronic Condition Distress scale), lower self-efficacy for managing chronic illness (PMCSM), and poorer general physical and mental health (PROMIS-10). Magnitudes of the majority of these correlations (84\%) were medium-size or greater (rho $\geq 0.30$ ) ${ }^{15}$ supporting convergent validity. Nine of eleven PETS domain scores were also significantly correlated with the medication convenience subscale of the TSQM, with higher PETS burden scores associated with lower perceived medication convenience ( $73 \%$ of correlations above 0.30 ). PETS domain scores were less consistently correlated with the medications side effects subscale of the TSQM (36\% of correlations above 0.30 ). The PETS domains most reflective of interactions with health care providers and services were significantly associated with a rating of convenience of health care services. Greater burden of medical appointments, medical and health care expenses, and difficulty with health care services from the PETS were all associated with lower ratings of convenience of health care services (two of the three correlations above 0.30). Finally, from patient data extracted from the electronic health record, the majority of PETS scores were uncorrelated with number of diagnosed conditions and number of recent encounters with health care providers (no correlations above 0.30 ). Only the PETS medications and interpersonal challenges domains were both significantly associated with the number of recent provider encounters; however, the magnitudes of these correlations were small (rhos $<0.30$ ).

\section{Known-groups comparisons of PETS scores}

Mean PETS scale scores were compared across meaningfully distinct patient groups as defined by self-reported medication adherence and financial difficulties. Compared to those who reported $100 \%$ adherence to provider recommended medications, those reporting less than $100 \%$ adherence reported significantly more treatment burden in eight of eleven PETS domains (Figure 1). Effect sizes of these differences were mostly medium-to-large in magnitude $(d>0.50$ for $82 \%$ of the group differences). Furthermore, compared to patients indicating no or only "a little" financial difficulty due to their medical treatment or physical condition, those indicating financial difficulty "somewhat or more" reported significantly greater treatment burden in ten of eleven PETS domains (all $p$-values $<0.01$, Figure $\mathrm{S} 2$ ). Effect sizes of these differences were also mostly medium-to-large in magnitude $(d>0.50$ for $91 \%$ of the group differences).

\section{Predictive ability of PETS domain scores}

Four forward-entry step-wise regressions were conducted (three linear and one logistic) to explore which PETS domain scores are most associated with distress, overall physical and mental health, and medication adherence, after controlling for number of comorbid conditions and number of provider encounters. In regression 1, greater distress as assessed by the Chronic Condition Distress scale was significantly associated with more role/social activity limitations due to self-management (standardized regression coefficient, referred to forthwith as beta $=0.39$ ), more medication burden (beta $=0.24$ ), more monitoring burden (beta $=0.23$ ), and more interpersonal challenges with other people (beta $=0.23)\left(F[6,67]=27.7, p<0.001\right.$; overall model $\left.\mathrm{R}^{2}=0.71\right)$. In regression 2, worse physical health as measured by the GPH score of the PROMIS-10 was significantly associated with greater difficulty with health care services (beta $=-0.32$ ), 
Table 2 Descriptive statistics and reliability of eleven PETS domain scales in diabetes patients

\begin{tabular}{llllll}
\hline PETS domain (no. of items) & N & Mean (SD) & Score range & Missing, \% & Cronbach's $\alpha$ \\
\hline Medical information (7) & 110 & $26.0(18.1)$ & $0-78.6$ & $8 \%$ & 0.89 \\
Medications (7) & 110 & $12.2(17.3)$ & $0-78.6$ & $8 \%$ & 0.89 \\
Medical appointments (3) & 109 & $11.0(18.7)$ & $0-83.3$ & $9 \%$ & 0.83 \\
Monitoring health (2) & 108 & $20.3(22.3)$ & $0-100$ & $10 \%$ & 0.74 \\
Interpersonal challenges (4) & 113 & $14.8(19.9)$ & $0-81.3$ & $6 \%$ & 0.81 \\
Medical and health care expenses (5) & 105 & $41.3(27.3)$ & $0-100$ & $13 \%$ & 0.93 \\
Difficulty with health care services (7) & 104 & $32.0(17.1)$ & $0-81.0$ & $13 \%$ & 0.82 \\
Role/social activity limitations (6) & 113 & $17.0(21.8)$ & $0-91.7$ & $6 \%$ & 0.93 \\
Physical/mental exhaustion (5) & 114 & $26.6(24.3)$ & $0-100$ & $5 \%$ & 0.92 \\
Bother due to reliance on medicine (I) & 111 & $24.5(31.1)$ & $0-100$ & $8 \%$ & NA \\
Bother due to medicine side effects (I) & 111 & $15.3(21.6)$ & $0-75$ & $8 \%$ & NA
\end{tabular}

Note: \#AIl PETS domain scores are standardized to a 0 (lowest burden) to 100 (highest burden) scale.

Abbreviations: SD, standard deviation; NA, not applicable; PETS, Patient Experience with Treatment and Self-Management.

Table 3 Spearman's correlations (rho) of PETS domain scores with other measures

\begin{tabular}{|c|c|c|c|c|c|c|c|c|c|}
\hline PETS domain $\#$ & $\begin{array}{l}\text { Chronic } \\
\text { condition } \\
\text { distress }\end{array}$ & $\begin{array}{l}\text { TSQM } \\
\text { side } \\
\text { effects }\end{array}$ & $\begin{array}{l}\text { TSQM } \\
\text { convenience }\end{array}$ & PMCSM & $\begin{array}{l}\text { PROMIS } \\
-10 \\
\text { physical } \\
\text { health }\end{array}$ & $\begin{array}{l}\text { PROMIS } \\
-10 \\
\text { mental } \\
\text { health }\end{array}$ & $\begin{array}{l}\text { H-C } \\
\text { services } \\
\text { rating" }\end{array}$ & $\begin{array}{l}\text { No. of } \\
\text { chronic } \\
\text { conditions }\end{array}$ & $\begin{array}{l}\text { No. of } \\
\text { provider } \\
\text { encounters }\end{array}$ \\
\hline Medical information & $0.56 * * *$ & $-0.28 * *$ & $-0.45 * * *$ & $-0.46 * * *$ & $-0.50 * * *$ & $-0.57^{* * *}$ & $-0.44 * * *$ & $0.03 \mathrm{NS}$ & $0.09 \mathrm{NS}$ \\
\hline Medications & $0.53^{* * *}$ & $\begin{array}{l}-0.22 \\
\text { NS }\end{array}$ & $-0.45 * * *$ & $-0.33^{* * *}$ & $-0.4 I^{* * *}$ & $-0.4 I^{* * *}$ & $-0.25 * *$ & $0.02 \mathrm{NS}$ & $0.26 * *$ \\
\hline Medical appointments & $0.44 * * *$ & $\begin{array}{l}-0.22 \\
\text { NS }\end{array}$ & $-0.19 N S$ & $-0.31 * *$ & $-0.40 * * *$ & $-0.39 * * *$ & $-0.29 * *$ & $\begin{array}{l}0.04 \\
\text { NS }\end{array}$ & $\begin{array}{l}0.14 \\
\text { NS }\end{array}$ \\
\hline Monitoring health & $0.50 * * *$ & $-0.29 * *$ & $-0.37 * * *$ & $-0.50 * * *$ & $\begin{array}{l}-0.20 \\
\text { NS }\end{array}$ & $-0.29 * *$ & $\begin{array}{l}-0.20 \\
\text { NS }\end{array}$ & $\begin{array}{l}-0.15 \\
\text { NS }\end{array}$ & $\begin{array}{l}0.19 \\
\text { NS }\end{array}$ \\
\hline Interpersonal challenges & $0.62^{* * *}$ & $\begin{array}{l}-0.21 \\
\text { NS }\end{array}$ & $-0.34 * * *$ & $-0.42^{* * *}$ & $-0.44 * * *$ & $-0.54 * * *$ & $\begin{array}{l}-0.21 \\
\text { NS }\end{array}$ & $\begin{array}{l}-0.06 \\
\text { NS }\end{array}$ & $0.23 *$ \\
\hline Health care expenses & $0.48 * * *$ & $-0.29 * *$ & $-0.40 * * *$ & $-0.39 * * *$ & $-0.4 I^{* * *}$ & $-0.40 * * *$ & $-0.33^{* * * *}$ & $\begin{array}{l}0.01 \\
\text { NS }\end{array}$ & $\begin{array}{l}0.19 \\
\text { NS }\end{array}$ \\
\hline Difficulty with health care services & $0.28 * *$ & $\begin{array}{l}-0.15 \\
\text { NS }\end{array}$ & $\begin{array}{l}-0.22 \\
\text { NS }\end{array}$ & $-0.27 * *$ & $-0.34 * * *$ & $-0.38 * * *$ & $-0.45 * * *$ & $\begin{array}{l}-0.09 \\
\text { NS }\end{array}$ & $\begin{array}{l}0.08 \\
\text { NS }\end{array}$ \\
\hline Role/social activity limitations & $0.62 * * *$ & $-0.34 * * *$ & $-0.44 * * *$ & $-0.37^{* * *}$ & $-0.50 * * *$ & $-0.36 * * *$ & $-0.24^{*}$ & $\begin{array}{l}0.12 \\
\text { NS }\end{array}$ & $\begin{array}{l}0.18 \\
\text { NS }\end{array}$ \\
\hline Physical/mental exhaustion & $0.56 * * *$ & $-0.37 * * *$ & $-0.5 \mathrm{I} * * *$ & $-0.47^{* * *}$ & $-0.45^{* * *}$ & $-0.52 * * *$ & $-0.24 *$ & $\begin{array}{l}0.03 \\
\text { NS }\end{array}$ & $\begin{array}{l}0.19 \\
\text { NS }\end{array}$ \\
\hline Bother reliance on medicine & $0.45^{* * *}$ & $-0.32 * * *$ & $-0.23^{*}$ & $-0.25^{*}$ & $-0.36 * * *$ & $-0.35 * * *$ & $\begin{array}{l}-0.18 \\
\text { NS }\end{array}$ & $\begin{array}{l}-0.05 \\
\text { NS }\end{array}$ & $\begin{array}{l}0.21 \\
\text { NS }\end{array}$ \\
\hline Bother side effects of medicine & $0.39 * * *$ & $-0.61 * * *$ & $-0.32 * * *$ & $-0.31 * *$ & $\begin{array}{l}-0.17 \\
\text { NS }\end{array}$ & $-0.23 *$ & $\begin{array}{l}-0.15 \\
\text { NS }\end{array}$ & $\begin{array}{l}-0.06 \\
\text { NS }\end{array}$ & $\begin{array}{l}0.05 \\
\text { NS }\end{array}$ \\
\hline
\end{tabular}

Notes: \#Higher PETS score = more burden. "Health care $(\mathrm{H}-\mathrm{C})$ services rating: "How would you rate the convenience of health care services that you seek for your health problems?" $\left(0=\right.$ not at all convenient to $10=$ extremely convenient). $* * * P<0.00 \mathrm{I}$ (I-tailed); $* * P<0.005$ (I-tailed); ${ }^{*} P<0.0 \mathrm{I}$ ( $\mathrm{I}$-tailed).

Abbreviations: PETS, Patient Experience with Treatment and Self-Management; TSQM, Treatment Satisfaction Questionnaire for Medication; PMCSM, Perceived Medical Condition Self-Management scale; PROMIS, Patient-Reported Outcomes Measurement Information System; NS, not significant.

more physical and mental exhaustion due to self-management (beta $=-0.31$ ), and more medication burden (beta $=-0.22$ ) $\left(F[6,67]=11.9, p<0.001\right.$; overall model $\left.\mathrm{R}^{2}=0.47\right)$. In regression 3, worse mental health as measured by the GMH score of the PROMIS-10 was significantly associated with more physical and mental exhaustion due to self-management (beta $=-0.36$ ), more burden with medical information (beta $=-0.34$ ), and more medication burden (beta $=-0.21$ ) $\left(F[5,66]=15.6, p<0.001\right.$; overall model $\left.\mathrm{R}^{2}=0.54\right)$. Finally, in regression 4, logistic regression was used to determine which PETS domain scores were most associated with patients' adherence to taking recommended medications. Poorer medication adherence was associated with more burden with medical information (odds ratio $=0.94,95 \%$ CI: 0.90 , 0.99 ) and more medication burden (odds ratio $=0.96,95 \%$ CI: $0.92,0.99)\left(\chi^{2}[4]=23.5, p<0.001\right.$ for final model $)$. Full results of these regressions can be found in Tables S1-S4). Among the covariates, only number of comorbid conditions 


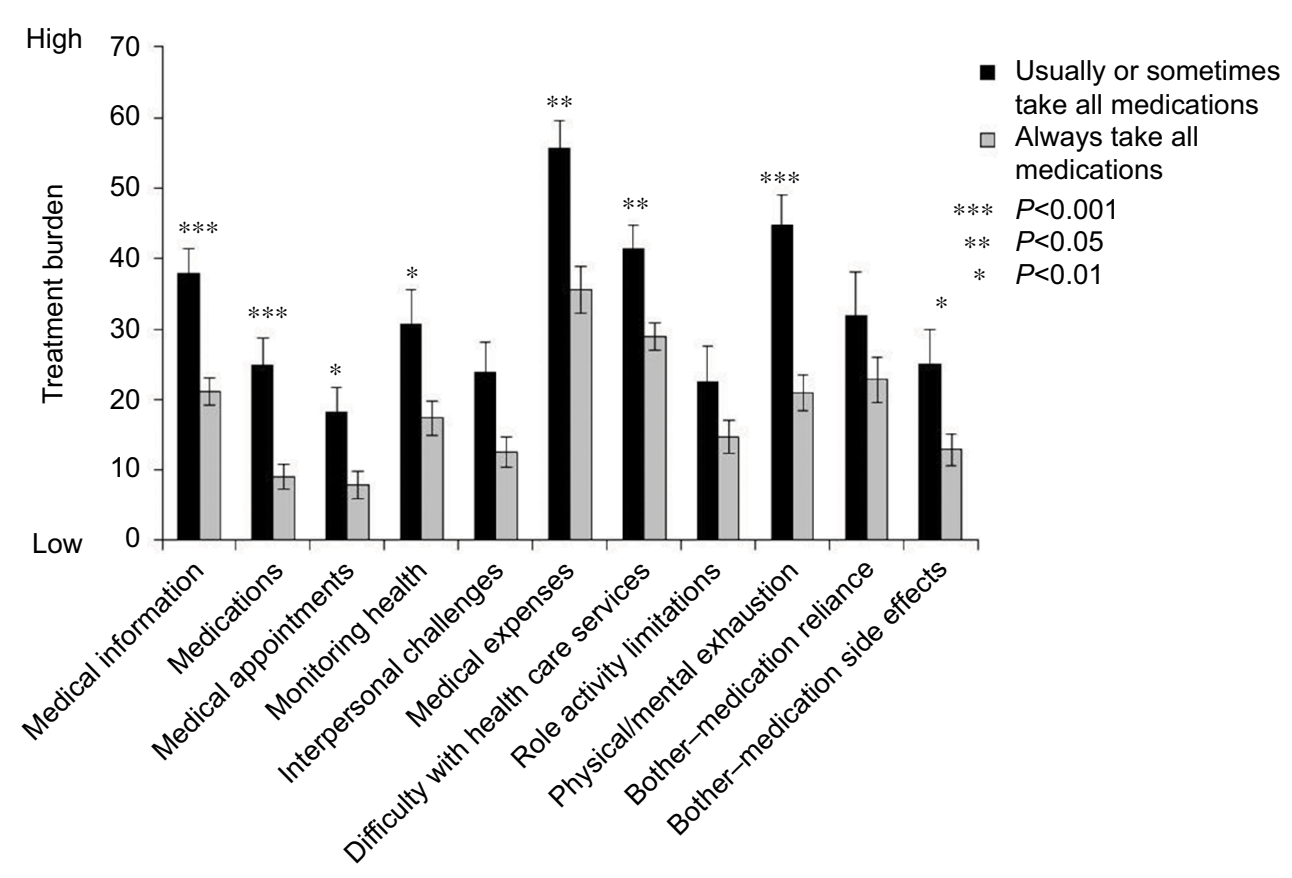

Figure I Mean PETS domain score comparisons by medication adherence, with standard error bars. Abbreviation: PETS, Patient Experience with Treatment and Self-Management.

was significantly associated with an outcome. Having more comorbid conditions was associated with reporting worse physical health (Table S2).

\section{Discussion}

In this study of 120 people with diabetes from two different health care settings, we found that domain scales of the PETS, a self-report measure of treatment burden, were correlated with established measures of distress, self-efficacy, and general physical and mental health. We found that mean PETS domain scores differentiate meaningful groups of diabetes patients based on self-reported medication adherence and financial difficulties due to medical treatment or health care, with most of these differences being medium-to-large in magnitude. Several PETS domains were also correlated with self-reported medication satisfaction (i.e., convenience and experience of side effects), and the medication burden scale was most consistently associated with well-being and adherence outcomes. Our findings support the reliability and validity of the PETS in people living with comorbid diabetes mellitus.

The ADA recommends that "providers should consider the burden of treatment [...] when making treatment recommendations", ${ }^{11}$ echoing recommendations for caring for patients with multimorbidity. ${ }^{30}$ Current treatment guidelines and clinical reimbursement structures tend to focus on individual diseases, which may inadvertently push clinicians to focus on disease-based metrics rather than on the problems that are of most concern to the patient. The need for patientreported measures to efficiently assess the patient's sense of treatment burden when managing complex chronic illness, including diabetes, is paramount. Adapting a self-report tool such as the PETS for a clinical setting could allow for patients to more efficiently communicate their struggles with treatment burden in a standardized way. This could then facilitate patient-clinician communication to move away from automatic attribution of suboptimal hemoglobin A1c to poor self-management behaviors, and bring the conversation into a non-judgmental realm that can focus on problem-solving and collaboration around the patient's holistic self-management support needs. Patient self-reporting of concerns would appear necessary as treatment and self-management burden does not appear to be easily gleaned from review of the medical record, as was demonstrated in this study.

Overall, there was some consistency in the association of PETS domains with distress, general physical and mental health, and medication adherence in this patient sample. Burden associated with taking medications (e.g., organizing, taking, and adjusting medications) was associated with all four of these self-reported outcomes. Perhaps more thorough efforts to educate diabetes patients about their medications, including how to take them, could be beneficial. Or, perhaps clinicians need to pay closer attention to reducing the number of medications that patients with diabetes are prescribed, 
working to find the best fit for patients and utilizing their understanding of available evidence to "deprescribe" lowvalue and burdensome medications as recommended in the Minimally Disruptive Medicine approach. ${ }^{31}$

The burden associated with finding, learning about, and understanding medical information and the perception of physical and mental exhaustion due to self-management were also associated with patient well-being and adherence. Identifying burden in these areas using the PETS could facilitate shared decision-making discussions between clinicians and patients, and inform potential solutions such as referrals to appropriate supportive services. Perhaps greater patient access to sources of trustworthy and understandable medical information could promote a sense of control over one's medical regimen and mitigate feelings of self-management burden. While these findings are suggestive, a more definitive test of the predictive validity of the PETS will require prospective data where burden domains are used to predict future patient outcomes such as hemoglobin A1c level.

Some limitations to our study exist. Though our data provide early evidence of reliability and validity in a diverse sample of patients with comorbid diabetes, its cross-sectional design is a limitation. Large-scale prospective testing of the PETS is underway that will determine its responsiveness to change, predictive validity, test-retest reliability, and the replicability of its factor structure. Alternative scoring algorithms will also be investigated (i.e., aggregated components of multiple domains) to reduce the number of scores derived from the measure. While the current version of the PETS is lengthy, this is offset by the unique contributions of its independently validated domain scales, each capturing important aspects of treatment burden and reducing the need for multiple questionnaires. Moreover, since there is no total burden score, users are free to select those PETS scales that are best suited to their study or clinical setting. We are currently working to develop shortened adaptations of the PETS, including for performance measurement of health care entities and a brief clinical tool for use with individual patients at the point-of-care.

Because the parent study was not designed as a diabetes study, we do not have available the proportion of each diabetes type in our cohort, though if our sample reflects the US population, it is likely that less than $6 \%$ had type 1 diabetes. ${ }^{32}$ Therefore, we cannot say whether the PETS is equally reliable and valid in each group. However, because of self-management overlap between type 1 and 2, and because the burdens reflected in the PETS items should apply to either, we do not expect there to be vast differences in how the PETS performs between them. It was beyond the scope of this study to compare the sensitivity of the PETS with diabetes-specific measures that assess aspects of treatment burden. Certainly head-to-head comparisons with established measures can elucidate the utility of a new measure in a given population. As this study features an analysis of a subsample of patients from a larger study of people with multi-morbidity, it was not designed specifically to assess or abstract diabetes-specific outcomes such as hemoglobin A1c or any other disease-specific marker. Understanding the unanticipated finding that treatment burden was not associated with number of comorbidities or patient encounters will require further exploration in future work. Finally, the smaller sample size relative to the parent validation study somewhat reduced statistical power.

The need for a focus on patient-reported measures to efficiently assess psychosocial well-being, including the patient's sense of treatment burden when managing a complex chronic illness such as diabetes, is increasingly recognized internationally. ${ }^{11,30}$ Using the PETS to not only identify the presence of treatment burden in general, but also to specify in which domains it is felt most acutely, could facilitate focused interventions that tailor self-management and/or psychosocial support to each patient.

\section{Acknowledgments}

This work was supported by the National Institute of Nursing Research of the National Institutes of Health under award number R21NR012984 (DT Eton, principal investigator). EA Rogers was supported through the UMN KL2 Scholars Career Development Program (National Center for Advancing Translational Sciences of the National Institutes of Health Award Number UL1TR000114). The content is solely the responsibility of the authors and does not necessarily represent the official views of the National Institutes of Health. The authors declare that they have no conflict of interest. If interested in utilizing the PETS measure, please contact Dr Eton at eton.david@mayo.edu.

We thank Ms Ann Harris and personnel in the Mayo Clinic Survey Research Center for formatting, distribution, and receipt of the survey battery. A Thakur has changed affiliation and is now at Wilder Research, Amherst H. Wilder Foundation, St. Paul, MN, USA.

\section{Disclosure}

The authors report no conflicts of interest in this work. 


\section{References}

1. Lin PJ, Kent DM, Winn A, Cohen JT, Neumann PJ. Multiple chronic conditions in type 2 diabetes mellitus: prevalence and consequences. Am J Manag Care. 2015;21(1):e23-34.

2. Magnan EM, Gittelson R, Bartels CM, Johnson HM, Pandhi N, Jacobs EA, Smith MA. Establishing chronic condition concordance and discordance with diabetes: a Delphi study. BMC Fam Pract. 2015;16:42.

3. Eton DT, Elraiyah TA, Yost KJ, et al. A systematic review of patientreported measures of burden of treatment in three chronic diseases. Patient Relat Outcome Meas. 2013;4:7-20.

4. Eton DT, Ramalho de Oliveira D, Egginton JS, Ridgeway JL, Odell L, May CR, Montori VM. Building a measurement framework of burden of treatment in complex patients with chronic conditions: a qualitative study. Patient Relat Outcome Meas. 2012;3:39-49.

5. Eton DT, Ridgeway JL, Egginton JS, et al. Finalizing a measurement framework for the burden of treatment in complex patients with chronic conditions. Patient Relat Outcome Meas. 2015;6:117-126.

6. Sav A, Whitty JA, McMillan SS, Kendall E, Kelly F, King MA, Wheeler AJ. Treatment burden and chronic illness: who is at most risk? Patient. 2016;9(6):559-569.

7. Anderson RT, Girman CJ, Pawaskar MD, et al. Diabetes medication satisfaction tool: a focus on treatment regimens. Diabetes Care. 2009; 32(1):51-53.

8. Brod M, Skovlund SE, Wittrup-Jensen KU. Measuring the impact of diabetes through patient report of treatment satisfaction, productivity and symptom experience. Qual Life Res. 2006;15(3):481-491.

9. Snoek FJ, Pouwer F, Welch GW, Polonsky WH. Diabetes-related emotional distress in Dutch and U.S. diabetic patients: cross-cultural validity of the problem areas in diabetes scale. Diabetes Care. 2000;23(9):1305-1309.

10. Boyd CM, Wolff JL, Giovannetti E, et al. Health care task difficulty among older adults with multimorbidity. Med Care. 2014;52 Suppl 3:S118-S125.

11. Young-Hyman D, de Groot M, Hill-Briggs F, Gonzalez JS, Hood K, Peyrot M. Psychosocial care for people with diabetes: a position statement of the American Diabetes Association. Diabetes Care. 2016;39(12):2126-2140.

12. Authors/Task Force Members; Ryden L, Grant PJ, Anker SD, et al. ESC Guidelines on diabetes, pre-diabetes, and cardiovascular diseases developed in collaboration with the EASD: the Task Force on diabetes, pre-diabetes, and cardiovascular diseases of the European Society of Cardiology (ESC) and developed in collaboration with the European Association for the Study of Diabetes (EASD). Eur Heart J. 2013;34(39):3035-3087.

13. Eton DT, Yost KJ, Lai JS, et al. Development and validation of the Patient Experience with Treatment and Self-management (PETS): a patient-reported measure of treatment burden. Qual Life Res. 2017;26(2):489-503.

14. Schoenberg NE, Leach C, Edwards W. "It's a toss up between my hearing, my heart, and my hip": prioritizing and accommodating multiple morbidities by vulnerable older adults. J Health Care Poor Underserved. 2009;20(1):134-151.

15. Vogeli C, Shields AE, Lee TA, Gibson TB, Marder WD, Weiss KB, Blumenthal D. Multiple chronic conditions: prevalence, health consequences, and implications for quality, care management, and costs. J Gen Intern Med. 2007;22 Suppl 3:391-395.

16. Lee PG, Cigolle C, Blaum C. The co-occurrence of chronic diseases and geriatric syndromes: the health and retirement study. J Am Geriatr Soc. 2009;57(3):511-516.
17. Schneider KM, O’Donnell BE, Dean D. Prevalence of multiple chronic conditions in the United States' Medicare population. Health Qual Life Outcomes. 2009;7:82.

18. Polonsky WH, Fisher L, Earles J, Dudl RJ, Lees J, Mullan J, Jackson RA. Assessing psychosocial distress in diabetes: development of the diabetes distress scale. Diabetes Care. 2005;28(3):626-631.

19. Atkinson MJ, Sinha A, Hass SL, Colman SS, Kumar RN, Brod M, Rowland CR. Validation of a general measure of treatment satisfaction, the Treatment Satisfaction Questionnaire for Medication (TSQM), using a national panel study of chronic disease. Health Qual Life Outcomes. 2004;2:12.

20. Wallston KA, Osborn CY, Wagner LJ, Hilker KA. The Perceived Medical Condition Self-Management Scale applied to persons with HIV/ AIDS. J Health Psychol. 2011;16(1):109-115.

21. Hays RD, Bjorner JB, Revicki DA, Spritzer KL, Cella D. Development of physical and mental health summary scores from the patient-reported outcomes measurement information system (PROMIS) global items. Qual Life Res. 2009;18(7):873-880.

22. Wallston KA, Rothman RL, Cherrington A. Psychometric properties of the Perceived Diabetes Self-Management Scale (PDSMS). J Behav Med. 2007;30(5):395-401.

23. Kobau R, Cui W, Zack MM. Adults with an epilepsy history fare significantly worse on positive mental and physical health than adults with other common chronic conditions-Estimates from the 2010 National Health Interview Survey and Patient Reported Outcome Measurement System (PROMIS) Global Health Scale. Epilepsy Behav. 2017;72:182-184

24. ahrq.gov [homepage on the Internet]. Agency for Healthcare Research and Quality. Surveys and Tools to Advance Patient-Centered Care. CAHPS; 2013. Available from: https://www.ahrq.gov/cahps/index.html. Accessed September 28, 2017.

25. Morris NS, MacLean CD, Chew LD, Littenberg B. The Single Item Literacy Screener: evaluation of a brief instrument to identify limited reading ability. BMC Fam Pract. 2006;7:21.

26. Schoenthaler A, Montague E, Baier Manwell L, Brown R, Schwartz MD, Linzer M. Patient-physician racial/ethnic concordance and blood pressure control: the role of trust and medication adherence. Ethn Health. 2014;19(5):565-578.

27. Fairclough DL, Cella DF. Functional Assessment of Cancer Therapy (FACT-G): non-response to individual questions. Qual Life Res. 1996;5(3):321-329.

28. Frost MH, Reeve BB, Liepa AM, Stauffer JW, Hays RD; Mayo/FDA Patient-Reported Outcomes Consensus Meeting Group. What is sufficient evidence for the reliability and validity of patient-reported outcome measures? Value Health. 2007;10 Suppl 2:S94-S105.

29. Cohen J. Statistical Power Analysis for the Behavioral Sciences. Hillsdale, NJ: Lawrence Erlbaum Associates; 1988.

30. nice.org [homepage on the Internet]. National Institute for Health and Care Excellence. Multimorbidity: clinical assessment and management. London, UK: National Institute for Health and Care Excellence; 2016. Available from: https://www.nice.org.uk/guidance/ng56. Accessed September 28, 2017.

31. Leppin AL, Montori VM, Gionfriddo MR. Minimally disruptive medicine: a pragmatically comprehensive model for delivering care to patients with multiple chronic conditions. Healthcare (Basel). 2015;3(1):50-63.

32. Menke A, Orchard TJ, Imperatore G, Bullard KM, Mayer-Davis E, Cowie CC. The prevalence of type 1 diabetes in the United States. Epidemiology. 2013;24(5):773-774. 


\section{Supplementary materials}

\section{DOMAIN/ITEM}

Medical information: How easy/difficult has it been to ...

(Responses: very easy, easy, neither easy nor difficult, difficult, very difficult, not applicable)

learn about your health problem(s)?

learn what foods you should eat to stay healthy?

find information on the medications that you have to take?

understand changes to your treatment plan?

understand the reasons why you are taking some medicines?

find sources of medical information that you trust?

understand advice from different health care providers?

Medications: How much of a problem has it been for you to ...

(Responses: not at all, a little, somewhat, quite a bit, very much)

organize your medicines?

take more than one medicine every day?

take your medicines several times each day?

refill your medicines?

adjust your medicines (including the amount, type, or time when you take it)?

take your medicines as directed?

plan your daily activities around your medicine schedule?

Single-item indicators of medication bother: How bothered have you been by ...

(Responses: not at all, a little, somewhat, quite a bit, very much)

how much you have to rely on your medicine(s)?

side effects of your medicine(s)?

Medical appointments: How much of a problem has it been for you to ...

(Responses: not at all, a little, somewhat, quite a bit, very much)

make or keep your medical appointments?

schedule and keep track of your medical appointments?

make or keep appointments with different health care providers?

Monitoring health: How much of a problem has it been for you to ...

(Responses: not at all, a little, somewhat, quite a bit, very much)

monitor your health behaviors, e.g., tracking exercise, foods you eat, or medicines you take?

monitor your health condition, e.g., weighing yourself, checking blood pressure, or checking blood sugar?

Interpersonal challenges: How bothered have you been by ...

(Responses: not at all, a little, somewhat, quite a bit, very much)

feeling dependent on others for your health care needs?

others reminding you to do things for your health like take your medicine, watch what you eat, or schedule medical appointments?

your health care needs creating tension in your relationships with others?

others not understanding your health situation?

Medical \& health care expenses: How easy/difficult has it been for you to ...

(Responses: very easy, easy, neither easy nor difficult, difficult, very difficult, not applicable)

plan for the future because of your medical expenses?

pay for healthy foods?

pay for all of your medical expenses?

pay for your medicines?

understand what is and what is not covered by your health insurance?

Difficulty with health care services: How much do you agree/disagree with the following?

(Responses: strongly agree, agree, disagree, strongly disagree, not applicable)

Have problems with different health care providers not communicating with each other about my medical care

Have to see too many different specialists for my health problem(s) or illness(es)

Have problems filling out forms related to my health care

Have problems getting appointments at times that are convenient for me

Have problems getting appointments with a specialist

Have to wait too long at my medical appointments

Have to wait too long at the pharmacy for my medicine

Figure SI (Continued) 
Role and social activity limitations: How much has your self-care interfered with your ...

(Responses: not at all, a little, somewhat, quite a bit, very much)

work (include work at home)?

family responsibilities?

daily activities?

hobbies and leisure activities?

ability to spend time with family and friends?

ability to travel for work or vacation?

Physical and mental exhaustion: How often did your self-care make you feel ...

(Responses: never, rarely, sometimes, often, always)

angry?

preoccupied?

depressed?

worn out?

frustrated?

Figure SI The 48-item version of the Patient Experience with Treatment and Self-management (PETS): a measure of perceived treatment burden.

Notes: Reproduced with permission from Eton DT, Yost KJ, Lai JS, et al. Development and validation of the Patient Experience with Treatment and Self-management (PETS): a patient-reported measure of treatment burden. Qual Life Res. 2017;26(2):489-503.13 With the exception of items in the "difficulty with health care services" domain, all items reference a recall time period of the past 4 weeks. No recall time period is used for the difficulty with health care services items. @ 2016 Mayo Foundation for Medical Education and Research. All Rights Reserved. All requests to use copies of this questionnaire should be addressed to DT Eton, eton.david@mayo.edu.

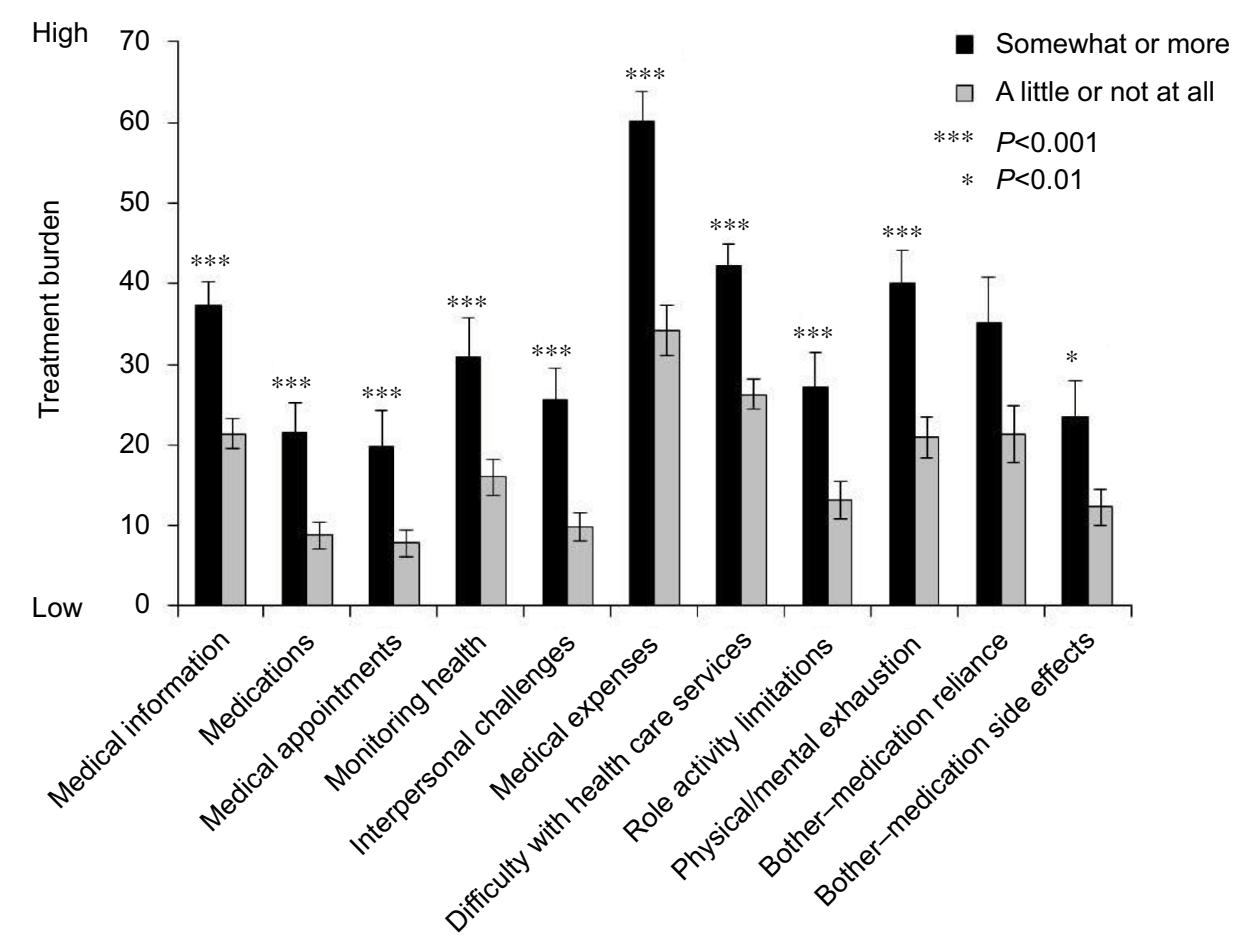

Figure S2 Mean PETS domain score comparisons by self-reported financial difficulties, with standard errors bars.

Abbreviation: PETS, Patient Experience with Treatment and Self-Management. 
Table SI Step-wise multiple regression of chronic illness distress onto PETS scores with covariates

\begin{tabular}{|c|c|c|c|}
\hline Regression models & B (unstandardized) & Standard error of $B$ & Beta (standardized) \\
\hline \multicolumn{4}{|l|}{ Step I } \\
\hline No. of comorbid conditions & -0.23 & 0.41 & -0.07 \\
\hline No. of provider encounters last 18 months & 0.06 & 0.03 & 0.23 \\
\hline \multicolumn{4}{|l|}{ Step 2} \\
\hline No. of comorbid conditions & -0.24 & 0.29 & -0.07 \\
\hline No. of provider encounters last 18 months & 0.00 & 0.02 & 0.02 \\
\hline PETS role/social activity limitations & 0.13 & 0.02 & $0.74^{* * *}$ \\
\hline \multicolumn{4}{|l|}{ Step 3} \\
\hline No. of comorbid conditions & 0.06 & 0.26 & 0.02 \\
\hline No. of provider encounters last 18 months & -0.01 & 0.02 & -0.04 \\
\hline PETS role/social activity limitations & 0.11 & 0.01 & $0.61^{* * *}$ \\
\hline PETS monitoring health & 0.07 & 0.02 & $0.36^{* * *}$ \\
\hline \multicolumn{4}{|l|}{ Step 4} \\
\hline No. of comorbid conditions & 0.10 & 0.25 & 0.03 \\
\hline No. of provider encounters last 18 months & -0.02 & 0.02 & -0.08 \\
\hline PETS role/social activity limitations & 0.09 & 0.01 & $0.52^{* * *}$ \\
\hline PETS monitoring health & 0.05 & 0.02 & $0.27^{* *}$ \\
\hline PETS medications & 0.06 & 0.02 & $0.27^{* *}$ \\
\hline \multicolumn{4}{|l|}{ Step 5} \\
\hline No. of comorbid conditions & 0.16 & 0.24 & 0.05 \\
\hline No. of provider encounters last 18 months & -0.03 & 0.02 & -0.09 \\
\hline PETS role/social activity limitations & 0.07 & 0.02 & $0.39 * * *$ \\
\hline PETS monitoring health & 0.04 & 0.02 & $0.23^{* *}$ \\
\hline PETS medications & 0.05 & 0.02 & $0.24 * *$ \\
\hline PETS interpersonal challenges & 0.05 & 0.02 & $0.23 *$ \\
\hline
\end{tabular}

Notes: Number of comorbid conditions and number of health care provider encounters in last 18 months are covariates, hence they are forced into all models. $R^{2}=0.05$ for step I. $\Delta R^{2}=0.50$ for step 2 ( $\left.p<0.00 I\right)$. $\Delta R^{2}=0.10$ for step 3 ( $\left.p<0.001\right) . \Delta R^{2}=0.05$ for step $4(p<0.005)$. $\Delta R^{2}=0.02$ for step 5 ( $p<0.05$ ). Final model (step 5) ANOVA: $F(6,67)=27.7, p<0.00$ I. Overall model $R^{2}=0.71$ (adjusted $\left.R^{2}=0.69\right)$. *** $p<0.001 ; * * p<0.01 ; * p<0.05$.

Abbreviation: ANOVA, analysis of variance; PETS, Patient Experience with Treatment and Self-Management.

Table S2 Step-wise multiple regression of PROMIS physical health onto PETS scores with covariates

\begin{tabular}{llll}
\hline Regression models & B (unstandardized) & Standard error of B & Beta (standardized) \\
\hline Step I & & & -0.11 \\
No. of comorbid conditions & -0.75 & 0.84 & -0.14 \\
No. of provider encounters last I8 months & -0.08 & 0.07 & -0.18 \\
Step 2 & & & -0.08 \\
No. of comorbid conditions & -1.20 & 0.71 & $-0.55^{* * *}$ \\
No. of provider encounters last I8 months & -0.41 & 0.06 & $-0.23^{*}$ \\
PETS difficulty with health care services & -0.24 & 0.04 & -0.01 \\
Step 3 & & & $-0.39^{* * *}$ \\
No. of comorbid conditions & -1.58 & 0.67 & $-0.37^{* *}$ \\
No. of provider encounters last 18 months & -0.00 & 0.05 & \\
PETS difficulty with health care services & -0.17 & 0.05 & $-0.24^{*}$ \\
PETS physical/mental exhaustion & -0.13 & 0.04 & 0.04 \\
Step 4 & & & $-0.32^{* *}$ \\
No. of comorbid conditions & -1.64 & 0.65 & $-0.31^{* *}$ \\
No. of provider encounters last I8 months & 0.02 & 0.05 & $-0.22^{*}$ \\
PETS difficulty with health care services & -0.14 & 0.05 & 0.04 \\
PETS physical/mental exhaustion & -0.11 & 0.05 & \\
PETS medications & -0.10 & & \\
\hline
\end{tabular}

Notes: Number of comorbid conditions and number of health care provider encounters in last 18 months are covariates, hence they are forced into all models. $R^{2}=0.04$ for step I. $\Delta R^{2}=0.29$ for step 2 ( $\left.p<0.00 I\right)$. $\Delta R^{2}=0.10$ for step $3(p<0.005) . \Delta R^{2}=0.04$ for step 4 ( $\left.p<0.05\right)$. Final model (step 4$)$ ANOVA: $F(6,67)=1 \mathrm{I} .9, p<0.00 \mathrm{I}$. Overall model $\mathrm{R}^{2}=0.47$ (adjusted $\mathrm{R}^{2}=0.43$ ). $* * * p<0.001 ; * * p<0.01 ; * p<0.05$.

Abbreviations: ANOVA, analysis of variance; PROMIS, Patient-Reported Outcomes Measurement Information System; PETS, Patient Experience with Treatment and Self-Management. 
Table S3 Step-wise multiple regression of PROMIS mental health onto PETS scores with covariates

\begin{tabular}{llll}
\hline Regression models & B (unstandardized) & Standard error of B & Beta (standardized) \\
\hline Step I & & & 0.02 \\
$\quad$ No. of comorbid conditions & 0.19 & 1.02 & -0.13 \\
$\quad$ No. of provider encounters last I8 months & -0.09 & 0.08 & -0.02 \\
Step 2 & & & -0.10 \\
$\quad$ No. of comorbid conditions & -0.16 & 0.80 & $-0.63^{* * *}$ \\
No. of provider encounters last I8 months & -0.07 & 0.06 & -0.08 \\
PETS medical information & -0.34 & 0.05 & -0.03 \\
Step 3 & & & $-0.43^{* * *}$ \\
No. of comorbid conditions & -0.65 & 0.74 & $-0.39 * * *$ \\
No. of provider encounters last 18 months & -0.02 & 0.06 & \\
PETS medical information & -0.23 & 0.05 & -0.09 \\
PETS physical/mental exhaustion & -0.16 & 0.04 & 0.03 \\
Step 4 & & & $-0.34^{* *}$ \\
No. of comorbid conditions & -0.74 & 0.73 & $-0.36^{* *}$ \\
No. of provider encounters last 18 months & 0.02 & 0.06 & $-0.21^{*}$ \\
PETS medical information & -0.18 & 0.06 & 0.04 \\
PETS physical/mental exhaustion & -0.15 & 0.06 & \\
PETS medications & -0.12 & & \\
\hline
\end{tabular}

Notes: Number of comorbid conditions and number of health care provider encounters in last 18 months are covariates, hence they are forced into all models. $R^{2}=0.02$ for step I. $\Delta R^{2}=0.39$ for step $2(P<0.00 I) . \Delta R^{2}=0.10$ for step $3(p<0.00 I) . \Delta R^{2}=0.03$ for step 4 ( $\left.p<0.05\right)$. Final model (step 4) ANOVA: $F(5,66)=I 5.6, p<0.00 I$. Overall model $\mathrm{R}^{2}=0.54$ (adjusted $\mathrm{R}^{2}=0.5 \mathrm{I}$ ). *** $p<0.001 ; * * p<0.01 ; * p<0.05$.

Abbreviations: ANOVA, analysis of variance; PROMIS, Patient-Reported Outcomes Measurement Information System; PETS, Patient Experience with Treatment and Self-Management.

Table S4 Step-wise logistic regression of medication adherence (100\% adherence) onto PETS scores with covariates

\begin{tabular}{|c|c|c|}
\hline Regression model & B (standard error) & Odds ratio $(95 \% \mathrm{Cl})$ \\
\hline \multicolumn{3}{|l|}{ Step I } \\
\hline No. of comorbid conditions & $0.37(0.27)$ & I.44 $(0.85,2.46)$ \\
\hline No. of provider encounters last 18 months & $-0.03(0.02)$ & $0.97(0.94,1.01)$ \\
\hline \multicolumn{3}{|l|}{ Step 2} \\
\hline No. of comorbid conditions & $0.38(0.3 \mathrm{I})$ & I.46 $(0.79,2.70)$ \\
\hline No. of provider encounters last 18 months & $-0.03(0.02)$ & $0.97(0.93,1.00)$ \\
\hline PETS medical information & $-0.08(0.02)^{* *}$ & $0.93(0.89,0.97)$ \\
\hline \multicolumn{3}{|l|}{ Step 3} \\
\hline No. of comorbid conditions & $0.39(0.33)$ & I.48 $(0.77,2.84)$ \\
\hline No. of provider encounters last 18 months & $-0.02(0.02)$ & $0.98(0.94,1.03)$ \\
\hline PETS medical information & $-0.06(0.03)^{*}$ & $0.94(0.90,0.99)$ \\
\hline PETS medications & $-0.04(0.02)^{*}$ & $0.96(0.92,0.99)$ \\
\hline
\end{tabular}

Notes: Number of comorbid conditions and number of health care provider encounters in last 18 months are covariates, hence they are forced into all models. Chi-square after step I: $\chi^{2}(2)=3.02$, not significant. Chi-square after step $2: \chi^{2}(3)=19.3, p<0.001$. Chi-square after step 3 (final model): $\chi^{2}(4)=23.5, p<0.001 .{ }^{* *} p<0.01 ; * p<0.05$.

Abbreviations: PETS, Patient Experience with Treatment and Self-Management; $\mathrm{Cl}$, confidence interval.

\section{Publish your work in this journal}

Patient Related Outcome Measures is an international, peer-reviewed, open access journal focusing on treatment outcomes specifically relevant to patients. All aspects of patient care are addressed within the journal and practitioners from all disciplines are invited to submit their work as well as healthcare researchers and patient support groups.

\section{Dovepress}

The journal is included in PubMed. The manuscript management system is completely online and includes a very quick and fair peer-review system. Visit http://www.dovepress.com/testimonials.php to read real quotes from published authors. 